\title{
INVESTIGATION OF EMERGING DRUG TARGETS FOR THE TREATMENT OF DEMENTIA
}

\author{
Eun-Mee Kim, School of Psychology, Ulster University, Cromore Road Coleraine, Co \\ Londonderry BT52 1SA, Northern Ireland; Eugene O'Hare, School of Psychology, \\ Queens University, 18-30 Malone Road, Belfast BT9 5BN, Northern Ireland.
}

Dementing diseases such as Alzheimer's disease are increasing in prevalence as advances in medical technologies extend life expectancy, yet there is no pharmacological intervention available to prevent, arrest, or reverse any of the dementing diseases. Pyrimidines and their derivatives have a wide range of pharmaceutical applications, and the pyrimidine template and its derivatives have antimicrobial (Moustafa et al., 2007), antifungal (Faty et al., 2015), antimalarial (Manohar et al., 2012), antiviral (Danesh et al., 2015), and antibacterial (Andrews and Ahmed, 2015) properties. Pyrimidines also have anti-inflammatory properties (Panda and Chowdary, 2008), and these may be of considerable interest with respect to combating the progression of Alzheimer's disease.

$A \beta$ toxicity is heavily implicated in Alzheimer's disease and therapeutic interventions have focused almost exclusively on the inhibition of this toxicity (e.g. Kayed et al, 2003; Tiraboschi et al., 2004; Shi et al., 2009). However, inflammation also plays an important role in Alzheimer's disease (see Kinney et al, 2018) and the identification of a compound possessing anti-toxicity and antiinflammatory properties might present a significant advance in pharmacological therapeutics. To this end, some work has already been conducted on 5-aryloxypyrimidines.

A pyrrolo[3,2-e][1,2,4]triazolo[1,5-a]pyrimidine dosedependently suppressed $A \beta(1-42)$-induced production of nitic oxide, TNF- $\alpha, I L-1 \beta$, and IL6, and reduced behavioural deficits in the rat (O'Hare et al., 2011). Scopes et al. (2012) reported a 5aryloxypyrimidine that bound to monomeric $A \beta(1-42)$, produced a blockade of $A \beta(1-42)$ aggregation, protected neuronal cell lines exposed to $A \beta(1-42)$, alleviated deficits in hippocampal LTP induced by exposure to $A \beta$ oligomers, and alleviated deterioration of learning and memory in the rat. Another 5-aryloxypyrimidine has been shown to protect against 7PA2 CM-induced deficits in synaptic plasticity and on learned behaviour in the rat (O'Hare et al. 2013), had a positive effect on cognition in APPPS1-21 transgenic mice (Lo et al. 2013), and lowered insoluble $A \beta$ levels and $A \beta$ plaque load in APPSL transgenic mice (Brunner et al., 2017). This compound was also found to reduce thioflavin- $T$ fluorescence, protect a neuronal cell line, and attenuate the $A \beta(1-42)$ effect on synaptophysin levels in mouse cortical neurons. Yet another 5-aryloxypyrimidine bound to monomeric $A \beta(1-42)$, protected neuronal cell lines exposed to $A \beta(1-$ $42)$, and reduced the deficit in in vivo LTP and complex behaviour (O'Hare et al., 2014). These findings suggest that 5aryloxypyrimidines present novel pharmacological candidates for the treatment of Alzheimer's disease and dementia.

\section{References}

Andrews B and Ahmed M (2015). An efficient synthesis, characterization and anti-bacterial activity of pyrimidine bearing 1,3,4-thiadiazole derivatives. Indian J Chem, 54B, 406-411. Bruner D, et al. (2017). SEN1500, a novel oral amyloid- $\beta$ aggregation inhibitor, attenuates brain pathology in a mouse model of Alzheimer's disease. Neurosci Lett, 660, 96-102.

Danesh A, et al. (2015). Antiviral activity evaluation of some pyrimidine derivatives using plaque reduction assay. J Chem Pharm Res, 7, 289-293.

Faty RM, et al. (2015). Microwave-assisted synthesis and antimicrobial evaluation of novel spiroisoquinoline and spiropyrido[4,3-d]pyrimidine derivatives. Molecules, 20,1842-1859. Kayed R, et al. (2003). Common structure of soluble amyloid oligomers impies common mechanism of pathogenesis. Science, 300, 486-489.

Kinney JW, et al. (2018). Inflammation as a central mechanism in Alzheimer's disease. Alzheimers Dement, 4, 575-590.

Lo AC, et al. (2013). Dose-dependent improvements in learning and memory deficits in APPPS1-21 transgenic mice treated with the orally active $A \beta$ toxicity inhibitor SEN1500. Neuropharmacology, 75, 458-466.

Manohar S, et al. (2012). Novel 4 aminoquinoline-pyrimidine based hybrids with improved in vitro and in vivo antimalarial activity. ACS Med Chem Lett, 3, 555-559.

Moustafa AH, et al. (2007). Synthesis of some new pyrimidine derivatives of expected antimicrobial activity. Phos Sulf Silic Rel Elem, 183, 115-135.

O'Hare E, et al. (2011). Novel anti-inflammatory compound SEN1176 alleviates behavioural deficits induced following bilateral intrahippocampal injection of amyloid- $\beta 1-42$. J Alz Dis, $25,219-229$. O'Hare E, et al. (2013). Orally bioavailable small molecule drug protects memory in Alzheimer's disease models. Neurobiol Aging, 34, 1116-1125.

O'Hare E, et al. (2014). Novel 5-aryloxypyrimidine SEN1576 as a candidate for the treatment of Alzheimer's disease. Int $\mathrm{J}$

Neuropsychopharmacol, 17, 117-126.

Panda SS and Chowdary PVR (2008). Synthesis of novel indolylpyrimidine antiinflammatory, antioxidant and antibacterial agents. Indian J Pharm Sci, 70, 208-215.

Scopes DIC, et al. (2012). A oligomer toxicity inhibitor protects memory in models of synaptic toxicity. Brit J Pharmacol, 167, 383392.

Shi C, et al. (2009). Protective effects of ginkgo biloba extract (EGb761) and its constituents quercetin and ginkolide $B$ against $\beta$ amyloid peptide-induced toxicity in SH-SY5Y cells. Chem-Biol Interact, 181, 115-123.

Tiraboschi $P$, et al. (2004). The importance of neuritic plaques and tangles to the development of and evolution of AD. Neurology, 62, 1984-1989. 